\title{
Nadie hablará de nosotras cuando hayamos muerto. Pérdida e invisibilidad del patrimonio artístico femenino
}

\author{
Teresa Alario Trigueros ${ }^{1}$; Laura Lucas Palacios ${ }^{2}$
}

Recibido: 27 de febrero de 2018 / Aceptado: 17 de mayo de 2018

Resumen. La actual LPHE, que entró en vigor en el año 1985, establece como principales objetivos el conocimiento, la conservación y la trasmisión del patrimonio cultural común. Independientemente de la evolución que ha vivido el concepto de patrimonio cultural en estos años, hay una parte de éste que sigue presentando especiales problemas: el generado por las mujeres a lo largo de la historia y hasta la actualidad.

En el caso de patrimonio artístico, en las más de tres décadas que han pasado desde la puesta en marcha de la Ley de Patrimonio la brecha de género en las instituciones museísticas no sólo no ha disminuido, sino que se ha hecho más amplia: se guardan, conservan y exponen un número de obras creadas por mujeres inferior al que correspondería si contrastásemos con la realidad de cada momento histórico, mientras se siguen adquiriendo muchas más obras de artistas hombres que de artista mujeres. Ello supone no sólo un grave problema para el concepto de patrimonio de nuestro presente, sino para el patrimonio que legaremos a las generaciones futuras, ya que parte de las obras que han tenido en el pasado o tienen actualmente un peso significativo en el panorama artístico tendrán más dificultades para conservarse.

Palabras clave: Patrimonio; Ley Patrimonio; arte femenino; museos; conservación; feminismo.

\section{[en] Nobody will talk about us when we are dead. Loss and invisibility of the feminine artistic heritage}

Abstract. The real LPHE, which entered into force in 1985, establishes as main objectives the knowledge, conservation and transmission of the common cultural heritage. Regardless of the evolution of the concept of cultural heritage in these years, there is a part that continues to present special problems: the one generated by women throughout history and up to the present.

In the case of artistic heritage, in the last three decades that have passed since the implementation of the Heritage Law, the gender gap in museum institutions has not only decreased, but has become broader: they are saved, they conserve and exhibit a number of works created by women inferior to that of correspondence and the contrast with the reality of each historical moment, while they continue to acquire more works by men artists of the women artist. This does not mean a serious problem for the concept of heritage of our present, but for the heritage that we will bequeath to future generations, that the parts of the works that have had in the past or currently have a significant weight in the artistic panorama more difficulties to conserve.

Keywords: Heritage; Heritage Law; feminine art; museums; conservation; feminism.

1 Departamento de Historia del Arte. Universidad de Valladolid

talario@arte.uva.es

Código ORCID: 0000-0002-0450-7373

2 Departamento de Didácticas de las Ciencias Experimentales, Sociales y Matemáticas. Universidad de Valladolid

laura.lucas.palacios@uva.es

Código ORCID: 0000-0002-7214-181X 
Sumario. 1. Introducción. 2. Patrimonio y equidad de género: marco legal en el estado español. 3. El patrimonio femenino: entre la pérdida y la invisibilidad. 4. Conclusiones.

Cómo citar: Alario Trigueros, T., Lucas Palacios, L. (2018) Nadie hablará de nosotras cuando hayamos muerto. Pérdida e invisibilidad del patrimonio artístico femenino, en Anales de Historia del Arte $\mathrm{n}^{\circ} 28$ (2018), 417-430.

\section{Introducción}

“Ser digno de estar en un museo significa merecer ser preservado de la desaparición”

Marian López Fernández $\mathrm{CaO}^{3}$

A pesar de los profundos cambios que se han producido en el concepto y la realidad del museo en las últimas décadas, hasta el punto de que María Bolaños ha hablado de la etapa de la "reinvención del museo", la presencia de las creaciones realizadas por las mujeres en el seno de las instituciones museísticas sigue teniendo grandes debilidades, presentando clamorosas ausencias y silencios ${ }^{5}$. Este problema se ha planteado recientemente en España, tanto desde instancias públicas como privadas.

Desde 2010, en que el Ministerio de Cultura puso en marcha el proyecto Patrimonio en Femenino, se ha venido reflexionando y haciendo propuestas de nueva praxis en torno a las aportaciones de las mujeres al patrimonio común y la necesidad de ofrecer una mirada más inclusiva, lo que implica la revisión del mismo concepto de Museo. La necesidad de revisar desde la óptica de género los Museos y el Patrimonio se reflejó también en el hecho de que ese mismo año la revista Her\&Mus dedicase un monográfico a este tema en su $\mathrm{n}^{\mathrm{o}}$ 3. Poco después la Asociación Mujeres en las Artes Visuales (MAV) publicaba Mujeres en el sistema del arte en España, una obra colectiva, en la que uno de los apartados se dedicaba a "Gestión y Patrimonio", en el que lógicamente se reflexionaba también sobre las instituciones museísticas.

Muchas son las cuestiones planteadas en estos años de reflexiones y experiencias, aunque éstas se resumen en el problema de la invisibilidad de las mujeres en los discursos canónicos de los museos, tanto públicos como privados, ya que "las narrativas dominantes en los museos no suelen poner en duda el canon" $"$ Y en estas narrativas la presencia de la obra femenina constituye un mero añadido:

3 López Fernández Cao, M. (2011). ¿Es necesario un museo de mujeres? Patrimonio en femenino, 77-82. Ministerio de Cultura: Madrid.

4 Bolaños, M. (1999). Historia de los museos en España. Gijón: Trea.

5 De este modo se tituló una de las muestras organizadas por el Ministerio de Cultura español en el contexto del proyecto Patrimonio en Femenino al que posteriormente nos referiremos.

6 Alario Trigueros, T. (2010). Sobre museos y mujeres. Un nuevo diálogo, Her\&Mus, 3, 19-24. 
en la mayor parte de las ocasiones cuando el/la visitante recorre las salas de los fondos permanentes de los grandes museos comprueba que el discurso hegemónico unitario y androcéntrico apenas si se ha matizado con la introducción de algunas piezas de artistas mujeres que, relegadas a un papel marginal, sirven para reforzar el papel del artista-varón como único héroe y verdadero protagonista de la historia del arte?

En lo que se refiere a los museos de artes visuales, y más allá de la cuestión de si "museos con mujeres" o "museos de mujeres" en que parece haberse centrado parte del debate durante unos años, existe un problema subyacente, que se ha atendido poco y que presenta una gran urgencia: el de la conservación y difusión de las creaciones artísticas femeninas como elementos patrimoniales. En gran parte de las ocasiones, la conservación de una obra pasa por su incorporación a los fondos museísticos (privados o públicos), depositarios de la tradición que, como afirma Griselda Pollock, es siempre selectiva: una versión del pasado intencionalmente selectiva de un pasado configurativo y un presente preconfigurado, que resulta entonces poderosamente operativo dentro del proceso de definición e identificación cultural y social ${ }^{8}$.

Llonch Molina y López Benito dicen que "los museos son las instituciones donde cada cultura reúne, expone y difunde aquel patrimonio que posee el valor suficiente como para cuidarlo, conservarlo y darlo a conocer" "Tener o no obra en los museos implica para el /la artista que su obra ha sido reconocida con el valor suficiente, que es reconocida como digna de formar parte de lo que una sociedad ha seleccionado para representarse a sí misma. Tras ese reconocimiento, en la línea de lo establecido en la Ley de Patrimonio española, tres son los objetivos que se platea el museo respecto a las obras que custodia y que citan Llonch Molina y López Benito (2010): cuidar, conservar y dar a conocer.

\section{Patrimonio y equidad de género: marco legal en el estado español}

Planteado el contexto, sería conveniente hacer una reflexión del propio concepto de patrimonio y la ley vigente en el estado español. La evolución del concepto de patrimonio, o lo que se entiende como tal en la actualidad, puede seguirse históricamente, a través del análisis de la legislación y documentación pública o privada que aporta información sobre los elementos que las sociedades en los diversos momentos históricos han considerado de una u otra forma relevantes, sobre los cuales se veía la necesidad de articular sistemas para su protección y conservación. Lógicamente tanto los elementos considerados como "patrimoniales" como los motivos por los que así han sido valorados socialmente han ido variando según la época histórica (Cuenca, 2003) $)^{10}$.

\section{Ibídem.}

8 G. Pollock. (2002). Disparar sobre el canon. Mora. Revista del Instituto Interdisciplinario de Estudios de Género, 8, 25-32.

9 Llonch Molina, N. y López Benito, V. (2010). La mujer, agente generador de nuevas realidades Museísticas. Her\&Mus, 3, 5-6.

10 Cuenca, J. M. (2003). El patrimonio en la didáctica de las ciencias sociales. Análisis de concepciones, dificultades y obstáculos para su integración en la enseñanza obligatoria (Tesis doctoral). Universidad de Huelva, Huelva. 
En España el auge del patrimonio, según Jiménez de Madariaga (2002) ${ }^{11}$, será paralelo a la formación del estado de las autonomías, reafirmándose las identidades culturales, así como medio para definir las singularidades y realzar la memoria histórica. Por ello el concepto se asociaba casi exclusivamente a los elementos relacionados con lo monumental, sobre todo bienes muebles y edificios significativos desde el punto de vista artístico y/o histórico. Elementos que generalmente eran representativos de una élite, sin valorarse los elementos significativos de las clases populares y, por otra parte, reflejo de una cultura androcéntrica. Esta concepción del patrimonio subyace en la actual legislación española LPHE (Ley 16/1985, de 25 de junio, del Patrimonio Histórico Español), que como su propio nombre indica, sigue identificando el Patrimonio con lo relacionado con la Historia. Resulta contradictorio que esta Ley, que inicialmente parece mirar más hacia el pasado, fuera aprobada en la década en que se abrieron más espacios museísticos y se generaron más colecciones de arte contemporáneo en ningún otro país, como afirman J.L. Marzo y P. Mayayo (2015) ${ }^{12}$ :

El número y tamaño de los museos y centros de arte moderno y contemporáneo abiertos en España a partir de los años ochenta no tiene equivalente en ningún país del mundo

Eran además los años en que las mujeres comenzaban a ser mayoría entre el alumnado de Bellas Artes, y también en los que algunas artistas como Ouka Lele o Patricia Gadea adquirían un gran éxito artístico y mediático como representantes de la llamada "movida madrileña". Se cruzaban dos elementos, contemporaneidad y género, que interferían con el concepto tradicional de patrimonio que subyacía en la ley. Sin embargo, lejos de atender estas demandas que la sociedad exigía, el concepto de patrimonio recogido en la ley que lo protege quedó anclado en el pasado.

A día de hoy, esta ley de patrimonio sigue vigente, aunque debería corregirse o completarse de facto en muchos de sus puntos, tras la aprobación de la Ley Orgánica 22/3/2007 para la Igualdad efectiva entre Mujeres y Hombres, que dedica un apartado al ámbito de la producción cultural y artística. Dicha ley, que en realidad viene a desarrollar lo que se dice en el artículo 14 de la Constitución Española, dispone en el artículo 26 que las autoridades públicas han de promover diversas actuaciones en el ámbito de sus competencias. Dos de estas resultan de especial interés para este tema:

- Adoptar iniciativas destinadas a favorecer la promoción específica de las mujeres en la cultura y a combatir su discriminación estructural y/o difusa.

- Promover la presencia equilibrada de mujeres y hombres en la oferta artística y cultural pública.

La última de las actuaciones de este artículo permite además adoptar "todas las acciones positivas necesarias para corregir las situaciones de desigualdad en la producción y creación intelectual artística y cultural de las mujeres". A pesar de que gran parte del articulado de esta ley orgánica ha tenido aún escaso efecto por la falta

11 Jiménez de Madariaga, C. (2002). La comercialización del Patrimonio Cultural. En VI Jornadas Andaluzas del Patrimonio Histórico. Sevilla: Consejería de Cultura de la Junta de Andalucía, 63-107.

12 Marzo, J.L. y Mayayo, P. (2015). Arte en España (1939-1915). Ideas, prácticas, políticas. Madrid: Cátedra. 
de implementación de medidas concretas y, en el caso de ser necesario, la consecuente asignación de recursos, lo cierto es que sitúa fuera de la legalidad gran parte de la gestión del patrimonio en la actualidad.

Es decir que el desarrollo de la Ley Orgánica 22/3/2007 para la Igualdad efectiva entre Mujeres y Hombres y su aplicación en el ámbito patrimonial, que aquí se trata, debería haber conllevado la revisión y relectura del patrimonio que se custodia en los museos, así como el cambio de la política de exposiciones y de adquisiciones -entre otras cuestiones- tal como se planteará más adelante. Así el "choque de legalidades" se ha convertido en un "choque de realidades" al convivir estas dos leyes, lo que ha generado polémicas y demandas significativas por parte de algunos sectores del mundo de la cultura, como se refleja en los informes que la Asociación Mujeres en las Artes Visuales (MAV) ha hecho públicos desde el año 2009, en los que se denuncia el flagrante incumplimiento de la Ley para la Igualdad efectiva entre Mujeres y Hombres en el ámbito de museístico y expositivo español. Así en el informe $\mathrm{n}^{0} 7$, realizado en 2011, sobre "Artistas españolas y sus obras en 10 museos de arte contemporáneo" se denunciaba que:

"la producción artística realizada por artistas españolas está diezmada en las colecciones de nuestros museos de arte contemporáneo y que sólo unas pocas obras se están guardando para legar al patrimonio español en el futuro"13.

Lo cierto es que, al hablar de patrimonio, estamos hablando también el concepto de ciudadanía que subyace y que ha de ser la base para entender el patrimonio; ya que no existiría el patrimonio sin una ciudadanía que se reconociera en él y viceversa. De hecho, este valor de generar ciudadanía está implícito en el valor que la propia LPHE da al patrimonio:

"Su valor lo proporciona la estima que, como elemento de identidad cultural, merece a la sensibilidad de los ciudadanos, porque los bienes que lo integran se han convertido en patrimoniales debido exclusivamente a la acción social que cumplen, directamente derivada del aprecio con que los mismos ciudadanos los han ido revalorizando" (Prólogo LPHE, 1985).

Debería, por tanto, garantizarse las fórmulas necesarias para que la ciudadanía en su conjunto pudiera verse representada, tener acceso a su herencia cultural y construir una identidad colectiva sin sesgos de género, raza, clase, religión etc.

Sin embargo, si echamos una mirada a las colecciones de los museos españoles vemos que en la actualidad los nombres femeninos entre los artistas que tienen obra en sus fondos son muy escasos y anecdóticos, siendo "natural la exclusión de las artistas mujeres de los grandes acontecimientos históricos"14 (Alario, 2010, p.20). De este modo, parece que lo valioso de nuestro patrimonio, de nuestro pasado, ha recaído únicamente en el género masculino, por lo que los elementos definitorios de nuestra

\footnotetext{
13 Informe MAV n ${ }^{\circ}$ 7. 18 de mayo de 2011. Web de MAV https: //mav.org.es/documentacion/informes/informemav-n7/ (fecha de consulta: 22 de mayo de 2018).

14 Alario Trigueros, M. T. (2010). Sobre museos y mujeres: un nuevo diálogo. Hermus, 3, 19-24.
} 
identidad cultural excluyen a la mitad de la ciudanía y, en el peor de los casos, definen una ciudadanía de mujer como objetos estéticos y de belleza supeditados al canon androcéntrico del momento histórico. Se invisibiliza así, todas las prácticas realizadas por y para mujeres y los bienes constitutivos de nuestra identidad, perpetuándose unos roles y unos modelos de actuación que han posibilitado que las sociedades patriarcales se asentaran plenamente. Tomando las palabras de López Fernández Cao (2011) ${ }^{15}$, podríamos decir que "la tradición ha sido guía para los hombres y carcelera para las mujeres y que ha creado un muro infranqueable de relaciones sociales estandarizadas y estereotipadas, difíciles, que no imposibles, de modificar".

Hall y Du Gay (2003) ${ }^{16}$ afirman que las identidades tienen que ver con las cuestiones referidas al uso de los recursos de la historia, la lengua y la cultura en el proceso de devenir y no de ser; no quiénes somos o de dónde venimos sino en qué podríamos convertirnos, cómo nos han representado y cómo atañe ello al modo como podríamos representarnos. Por su parte, Lowenthal (1998) ${ }^{17}$ afirma que el pasado es parte de nuestra identidad, porque la seguridad del yo era es un componente necesario para la seguridad del yo soy, de tal forma que la capacidad para recordar nuestro pasado e identificarnos con él ofrece significado, finalidad y valor a nuestra existencia.

Pero todos/as y cada uno/a de nosotros somos en realidad resultado de un cruce de identidades: de cultura, clase, etnia y de género. La cuestión de la interseccionalidad en la identidad es un tema que no podemos olvidar al hablar de patrimonio. El concepto monolítico de sujeto, androcéntrico y racional, que tiene su origen en la modernidad ha desaparecido. Y en este momento es cuando parece pertinente preguntarse por las identidades de género a la hora de conceptualizar el patrimonio. La idea no es hacer una "historia paralela" a partir de incorporar las creaciones de las mujeres a la narrativa existente, sino cuestionar a quien se ve como sujeto de la Historia. Todo ello pasa por "reposicionar" nuestra mirada, cuestionar el canon dado. Porque "ese canon patriarcal y androcéntrico se ha convertido en un techo de cristal blindado que limita las posibilidades de las artistas (...) en las instituciones museísticas, de modo que solo les está permitido avanzar hasta determinadas cotas" $(\text { Alario, 2011) })^{18}$.

En todo caso, lo que es una realidad evidente es que los museos y la museología no pueden vivir de espaldas a los retos que el siglo XXI les presenta, sino que ha de dar respuesta a los interrogantes que la sociedad se hace sobre la necesidad de concebir el patrimonio como una realidad integral capaz de asumir cualquier dimensión que afecte al ser humano en su totalidad (Hernández Hernández, 2007) ${ }^{19}$. En palabras de Irina Bokova, directora general de la Unesco "existe un reconocimiento creciente del papel de la cultura en la promoción del desarrollo social inclusivo, donde la igualdad de género es una parte fundamental de la ecuación para un desarrollo más inclusivo y sostenible" (Bokova, 2015) ${ }^{20}$.

\footnotetext{
15 López Fernández Cao, M. (2011). “¿Es necesario un museo de mujeres?” Patrimonio en femenino, 77-82. Ministerio de Cultura: Madrid.

16 Hall, S. y du Gay, P. (2003). Cuestiones de identidad cultural. España: Amorrotu Editores.

17 Lowenthal, D. (1998). El pasado es un país extraño. Madrid: Akal.

18 Alario Trigueros, T. (2011). Una mirada desde el género. Figuras de la exclusión, 127-138.

19 Hernández Hernández (2007). La Museología ante los retos del siglo XXI. E-rph. Revista Electrónica de patrimonio histórico, 1. Obtenido de: http: //revistaseug.ugr.es/index.php/erph/article/view/3326.

20 Bokova, I. (2015). Igualdad de género: patrimonio y creatividad, Argentina: Unesco.
} 
Por ello es necesario revisar y releer las colecciones de los museos e introducir las obras de mujeres artistas coprotagonistas de la historia, que han aportado miradas diferentes en todos los momentos históricos y que, a pesar de la evidente calidad de sus creaciones, hasta ahora han sido desconocidas. Para ello, el primer obstáculo que hay que resolver es localizar su obra y que las administraciones públicas encargadas de la gestión del patrimonio cumplan con lo establecido en el art. 46 de la Constitución, poniendo en marcha los mecanismos de gestión que le son otorgados como es conocer, planificar, controlar y difundir...las obras de hombres y mujeres de modo equitativo. Las cuatro tareas están conectadas entre sí y el cumplimiento de las mismas desde los organismos públicos gestores del patrimonio, facilitaría que los museos puedan cumplir con las funciones que la propia definición de la LPHE les otorga en su definición y que hemos recogido en líneas anteriores: adquirir, conservar, investigar, comunicar o exhibir.

\section{El patrimonio femenino: entre la pérdida y la invisibilidad}

En la última década se han desarrollado en el estado español interesantes proyectos cuyo eje fundamental -o al menos uno de ellos- era potenciar la igualdad de género en los museos. Cabe destacar proyectos como Relecturas. Itinerarios museales en clave de género, el Programa Reset, Museos más Sociales o Museos en Femenino.

Relecturas es un proyecto que tiene como objetivo acercar las colecciones de seis museos valencianos atendiendo a la perspectiva de género. Como bien define el título del proyecto el objetivo es releer los fondos de estos museos -que van de las Bellas Artes a la Etnografía o la Historia- desde la clave de género, de modo que las piezas se reactivan, generando nuevos discursos. El plan del Ministerio de Educación, Cultura y Deporte Museos Más Sociales nace contando con los 16 museos de la Dirección General de Bellas Artes y Bienes Culturales y de Archivos y Bibliotecas, a los que enseguida se incorporan otros 4 dependientes de la Secretaría de Estado de Cultura. Alguno de los museos que participan en este plan -como el Museo Nacional de Cerámica, el Arqueológico Nacional y el Museo Nacional Reina Sofía- formaban parte también de otros proyectos que inciden en la búsqueda de la igualdad de género como el arriba citado de Relecturas o de otros de los que se hablará más adelante (Didáctica 2.0 Museos en femenino). El objetivo de este plan es afrontar los nuevos retos sociales que tienen los museos, entre los que interesa destacar en este caso el fomento de la perspectiva de género. En ambos proyectos el compromiso de los museos - con perfiles y fondos muy diferentes- pasa por releer sus fondos desde el punto de vista de género y proponer exposiciones temporales y/o itinerarios específicos en los que se pone el foco sobre las mujeres en distintos momentos de la historia y diferentes actividades. Pero lo cierto es que el cumplimiento de los objetivos de la transversalidad de género ha sido muy diferente en cada uno de los casos, tratándose en algunos casos más un deseo que una realidad.

De todos los proyectos citados cabe destacar Didáctica 2.0 Museos en femenino. Este proyecto fue desarrollado por un equipo formado por varias investigadoras del Instituto de Investigaciones Feministas de la Universidad Complutense de Madrid, coordinados por las profesoras Marián López F. Cao, Antonia Fernández Valencia y Asunción Bernardez Rodal, la Asociación e-Mujeres y los equipos de los Museos participantes. El proyecto se centró en las mujeres en el arte, participando, entre 
otros los grandes museos nacionales de Madrid: Museo Arqueológico Nacional, Museo del Pardo y Museo Nacional Reina Sofía. Uno de los resultados más visibles de este proyecto fue, como en los proyectos arriba citados, el diseño de itinerarios específicos en los que se releía parte de los fondos de desde la óptica de las teorías feministas y de género. Desde el punto de vista de la visibilidad de las mujeres artistas y sus obras es especialmente interesante el itinerario que, en el marco de este proyecto, se propuso en Museo Nacional Reina Sofía, titulado "Una mirada feminista desde las vanguardias".

Por tanto, se han dado pasos en buena dirección, aunque generalmente el proceso ha comenzado por los "bordes" incorporando actividades puntuales, conferencias o exposiciones temporales que visibilizan a las mujeres en relación con los fondos específicos de cada museo. Un paso más lo han dado aquellos museos que han diseñado itinerarios o recorridos de sus fondos partiendo de una visión de género como sucede con los museos que participaron en el proyecto Didáctica 2.0 Museos en femenino.

Otro proyecto de interés que se ha desarrollado a través de internet es Patrimonio en Femenino, consistente en una "exposición virtual organizada por la Dirección General de Bellas Artes y Bienes Culturales del Ministerio de Cultura que analiza desde una perspectiva de género la presencia y participación activa de las mujeres a lo largo de la historia, tanto en el ámbito público como privado, a través de un recorrido entre las colecciones de treinta museos españoles integrantes de la Red Digital de Colecciones de Museos de España"21

Podrían citarse otros ejemplos de museos que, con el objetivo de la igualdad de género, han desarrollado proyectos más o menos puntuales como ¿Quién da la vuelta a la tortilla? en el que a lo largo de 2011 y 2012 se implicaron los museos de Lugo, León y Murcia. El objetivo de este artículo, sin embargo, no es analizar -ni siquiera citar de un modo exhaustivo no organizado cronológicamente- todas las experiencias que ha habido en los diferentes museos del estado español. La intención es sólo recordar que, a pesar de que evidentemente se han hecho intentos por hacer de los fondos de los museos un elemento patrimonial más equitativo desde el punto de vista de género, un patrimonio en el que se reconozca toda la ciudadanía, aún quedan muchos pasos por dar para llegar a la meta de la equidad de género. Equidad de género que es un concepto que va más allá de la igualdad, ya que parte una realidad evidente: la infrarrepresentación de las obras de creadoras mujeres en los museos que, como más adelante se verá no sólo no se ha paliado durante los últimos años, sino que ha ido en aumento.

Lo cierto es que como consecuencia de estos y otros proyectos la preocupación por visibilizar la obra de las artistas ha llegado a las grandes instituciones museísticas de artes plásticas y visuales del país, de modo que las obras de María Gutierrez Blanchard, Maruja Mallo o Ángeles Santos, entre otras, cuelgan en un lugar preeminente de sus salas como parte de la exposición permanente. Pero también es cierto que las creaciones de otras artistas importantes de nuestro inmediato pasado, como Remedios Varo o Delhy Tejero - que formaron parte de los mismos círculos de vanguardia y que tienen una obra interesante y muy personal- no están sin razón

${ }^{21}$ Web Patrimonio en femenino: https: //sede.educacion.gob.es/publiventa/detalle.action?cod=14187C (fecha de consulta: 23 de mayo de 2018). 
que lo justifique. Ausencias inexplicables si se quiere ofrecer un panorama ajustado a la realidad de lo que fue la presencia de las mujeres en la vanguardia española del primer tercio del siglo XX. Ausencias también peligrosas porque en algunos casos, al no hacerse cargo instituciones y museos de las obras, estas corren el riesgo de dispersarse 0 , incluso, de perderse.

Si miramos en el escalón inferior al que ocupan los grandes museos, en los de ámbito provincial, por ejemplo, este problema se hace más evidente: la ausencia de las obras de mujeres artistas es clamorosa y a ella se une el silencio que va un paso más allá, porque como afirma Gutiérrez Husillo (2012) "el silencio consiste en la omisión de algo. En este caso, el papel de la mujer ha estado presente, pero se relega o se borra". El primer recurso para borrar es muy sencillo: no nombrar. No podemos olvidar que "el procedimiento de exclusión más eficaz está en el núcleo de las propias narrativas culturales dominantes. Narrativas androcéntricas que reflejan la historia de la cultura como una historia de los logros del hombre-genio, y que ha estado ciega a la presencia de las mujeres como género en una historia construida desde postulados patriarcales" (Alario, 2011) ${ }^{23}$. Y si las autoras son borradas, en las siguientes generaciones difícilmente se conservarán sus obras. Un buen ejemplo son las artistas de finales del siglo XIX y principios del XX a quienes Estrella de Diego $(2009)^{24}$ definió como las tías-abuelas:

Uno de los mayores problemas que hay que afrontar a la hora de estudiar a las mujeres de todos los países y en todas las épocas es la desaparición de su evidencia. Descuido o malintencionado descuido (...) esconden la evidencia, la única prueba de que, además de ser hijas, esposas, madres, hermanas, amantes de pintores, las mujeres pintaron (...) Existen museos provinciales, donde se custodia hasta lo más pequeño en ese respetable afán regionalista, pero incluso allí los cuadros de las mujeres son escasos (...) están luego las colecciones privadas (...) la complicación aumenta porque para revalorizarlas es preciso librarse de los cuadros de las "tías-abuelas". Sólo el sentimentalismo salva las obras de las pintoras y cada vez resulta más difícil encontrar sentimentales.

Eso explica que en la investigación sobre las obras de las mujeres en las instituciones museísticas españolas que realizó Vicent Ibiza, titulada Artistas españolas antes del 36, sólo se recogen 275 nombres con obra en 66 museos que, además, tienen una escasa presencia en cuanto al $\mathrm{n}^{\circ}$ de piezas: 1,7 de media por artista.

El primer peligro es, por tanto, que las obras de las mujeres tienen más posibilidades que las de los hombres de perderse en los desvanes o dispersarse porque son custodiados en menor número de ocasiones en los museos. La pregunta que aún se hacen algunos es: ¿no están porque las hemos olvidados o porque no "tienen calidad suficiente" para estar? La pregunta se responde sola si vemos las investigaciones que en el caso del arte español se han venido haciendo desde que se publicó el libro de Estrella de Diego La mujer y la pintura del S. XIX español. Cuatrocientas olvidadas

22 Gutierrez Husillo, A. (2012). Meandros de la memoria. Ausencias y silencios en torno al patrimonio en femenino. Ausencias y silencios, 14-24.

23 Alario Trigueros, T. (2011). Una mirada desde el género. Figuras de la exclusión, 127-138.

24 Diego, E. (2009): La mujer y la pintura del S. XIX español. Cuatrocientas olvidadas y alguna más. Madrid: Cátedra. 
y alguna más. Nombres de artistas del siglo XIX y comienzos del XX como los de Lluisa Vidal, María Roësset, la vallisoletana Marcelina Porcela o la palentina María Romero, por poner algunos ejemplos, se han ido descubriendo en investigaciones posteriores, a veces incorporándose a pie forzado en una narrativa aun claramente androcéntrica. Pero en la mayor parte de los casos sus obras siguen dispersas y sin formar parte de los fondos de las instituciones museísticas.

En estos casos el problema se va agravando con el tiempo porque "el desinterés por la obra de las artistas ha dado lugar, además, a un problema añadido para la investigación actual, ya que se ha ido perdiendo parte de la información que proporciona documentación generada en el ámbito privado, como cartas, escritos, documentación gráfica, etc. que podía aportar un testimonio de primera mano" (Alario, 2006) ${ }^{25}$. Por otra parte, no era habitual en España que las artistas escribiesen memorias o diarios, como sucedía más allá de nuestras fronteras, lo que nos priva nuevamente de una fuente primordial y directa de información. No puede extrañar, por tanto, que todavía en la actualidad se den situaciones como la de una obra de Marcelina Porcela, que en la actualidad se expone en el Ayuntamiento de Valladolid con una cartela en que se lee "Marcelino Porcela"26. Es decir, no contentos con olvidar su obra, en algún momento se negó su sexo y el error no ha sido corregido hasta la actualidad.

Un ejemplo paradigmático de las graves consecuencias que la desmemoria puede tener para el patrimonio artístico generado por las mujeres es el de Delhy Tejero, cuya obra está entre las más más innovadoras del panorama artístico español durante el primer tercio del siglo XX. Delhy Tejero llegó a tener en la década de 1990 un museo dedicado a su obra en Toro (Zamora), su pueblo natal, pero sólo sobrevivió unos años. En la actualidad sus descendientes buscan una solución para que la obra de esta magnífica artista no se disperse y se pierda. El mismo hecho de un museo que lleve el nombre de una artista es aún una extrañeza en nuestro país, aunque nos parece normal hablar del Museo Picasso en Málaga o de la IAAC Pablo Serrano de Zaragoza.

En algunos casos al problema del género se une el de una coyuntura histórica que impactó especialmente en las trayectorias vitales de algunas generaciones de mujeres: la guerra civil y el triunfo del franquismo. Manuela Ballester o Victorina Durán, dos magníficas dibujantes, son ejemplo de las creadoras que tuvieron que exiliarse huyendo del régimen franquista. Por citar la obra de otras artistas cuya obra difícilmente pueden ver los/as visitantes de los museos españoles podemos referirnos a una pintora, como María Dapena, que sobrevivió al franquismo, pero cuya obra ha sido olvidada.

También durante la etapa franquista destacaron otras creadoras cuya obra no ha sido valorada posteriormente y no está conservada en museos como merecería. En este caso están algunas artistas que vivieron bajo el primer franquismo que podríamos decir que forman parte no de una sino de varias "generaciones perdidas". Un ejemplo es el de la pintora Magda Bolumar, una artista nacida en 1936 en Caldas de Estrach (Barcelona) a quien pocas personas recuerdan como algo más que la viuda del gran escultor informalista Moisés Villelia. Sin embargo, tanto la artista como su obra estuvieron fuertemente vinculadas a las vanguardias catalanas de las décadas

25 Alario Trigueros, T. (2006). María Paz Romero: La ilusión del éxito. En Alario, T. y García Colmenares, C. (Eds). Intelectuales palentinas del siglo XX. Palencia: Cálamo.

26 Cid, M. D. (2017). Marcelina Porcela (1864-1917). Una pintora en su contexto (Tesis doctoral). Universidad de Valladolid, Valladolid. 
centrales del siglo $\mathrm{XX}^{27}$. Magda Bolumar fue especialmente conocida por sus obras que tituló "Xarpelleres" y que expuso por vez primera en el año 1960 en varias salas de Barcelona. Estas obras tienen un sentido esencialmente constructivista, pues a pesar del papel innegable que en ellas tiene la materia y la textura, que las conectan en cierto modo con el Informalismo, la estructura adquiere el protagonismo principal a partir de la tensión de los hilos, tal como destacaba en varios textos el crítico Cirici Pellicer (Alario, 2015) ${ }^{28}$. Con una trayectoria reconocida a nivel nacional hasta la década de 1970, sin embargo, su obra no se conserva en ningún museo de carácter autonómico o nacional. Solo la voluntad de la propia artista y de sus descendientes, que están haciendo un gran esfuerzo de ordenación y catalogación de la obra realizada a lo largo de su vida, facilitará que esta le sobreviva. Al analizar el ejemplo de la obra de Magda Bolumar viene a la mente la afirmación de Gutiérrez Husillo (2012): "La ausencia es fácil de detectar, pero sólo cuando se es consciente de su existencia, es entonces cuando emerge con toda la fuerza que contiene, pero hasta entonces pasa totalmente desapercibida" 29

Desgraciadamente este caso no es una excepción. En el informe elaborado por la asociación Mujeres en las Artes Visuales (MAV) en el año 2011, titulado Artistas españolas y sus obras en 10 museos de arte contemporáneo, se destacaba que "La producción artística realizada por artistas españolas está diezmada en las colecciones de nuestros museos de arte contemporáneo y que sólo unas pocas obras se están guardando para legar al patrimonio español en el futuro" ${ }^{30}$. En datos tomados de los citados 10 museos destacaba el hecho de que porcentaje de obra de mujeres artistas en sus fondos no superaba en ningún caso el $20 \%$, siendo la media del conjunto el $13 \%$. Si tenemos en cuenta que estamos hablando de arte producido en los siglos XX y XXI, un período en que el número de mujeres en el panorama artístico español se multiplica exponencialmente, resulta llamativo que la presencia de las creaciones de las mujeres no "lleguen" a los museos, no se consideren dignas de ser seleccionadas como patrimonio común y, por tanto, expuestas, conservadas y divulgadas. Un buen ejemplo de los vacíos clamorosos en los fondos permanentes de los museos es el referido a la obra de la pintora Patricia Gadea, de quien se ha escrito que es un "personaje fundamental en la renovación de la pintura española de las décadas de los ochenta y noventa" ${ }^{31}$. Gadea formó parte de Strujenbank, uno de los colectivos más importantes de las dos últimas décadas del siglo XX desde el que hizo un crítica ácida y mordaz al modelo cultural promovido oficialmente, especialmente en su serie Circo. Tras la disolución de Strujenbank y hasta su muerte, en el año 2006, Patricia Gadea siguió pintando con los códigos heredados de etapas anteriores, pero con una visión crítica más cercana al feminismo. A pesar de que estas obras pudieron verse y reivindicarse en la exposición que el Museo Nacional Reina Sofía le dedicó a finales

27 Estuvo vinculada al grupo de Arte Actual de Mataró y más indirectamente con algunos artistas de Dau al set, colaboró estrechamente con la revista Inquietud artística, manteniendo amistad con Joan Brossa y Cirici Pellicer, entre otros.

28 Alario Trigueros, T. (2015). Aracne en el arte contemporáneo español. En Puleo, A. (Eds.), Hacia Una cultura de la sostenibilidad. Análisis y propuestas desde la perspectiva de género, 251-262. Valladolid: Universidad de Valladolid.

29 Gutiérrez Husillo, A. (2012). Meandros de la memoria. Ausencias y silencios en torno al patrimonio en femenino. Ausencias y silencios, 14-24.

30 VV.AA. (2012). Mujeres en el sistema del arte español en España. MAV/EXIT: Madrid.

31 Catálogo de la exposición Atomic Circus. Museo Nacional Reina Sofía. 2014. 
del año 2014, este período de su obra está muy escasamente representada en nuestras instituciones museísticas.

Los ejemplos citados de creadoras del siglo XX son sólo algunos de los nombres que, por derecho propio y por la calidad de sus obras, deberían incorporarse al acervo común del patrimonio que se custodia, conserva y muestra en nuestros museos.

Uno de los problemas de la escasa representatividad de las artistas en el ámbito de los museos es que las nuevas adquisiciones que estos realizan están claramente desequilibradas a favor de las obras creadas por los artistas varones: El informe de MAV de 2014 lo ponía claramente en evidencia al destacar que el 85\% de las nuevas adquisiciones de los museos eran de hombres y sólo el 15\% de mujeres. Podríamos afirmar, por tanto, que desde el punto de vista cuantitativo la brecha de género en los museos se ha ido ampliando en vez de disminuir a pesar de las declaraciones de búsqueda de la equidad que impregnan el discurso oficial. Hay que destacar este hecho porque se produce justamente en un período histórico en el que, como se ha destacado anteriormente, existe una normativa legal que vela por la igualdad entre mujeres y hombres en todos los ámbitos, entre los que está la cultura (Ley de Igualdad. Ley Orgánica 22/3/2007). Esta es la dura realidad a pesar de que en la programación temporal de los museos se haya intentado compensar con exposiciones monográficas o colectivas sobre las trayectorias de diversas artistas.

Al hecho de que no están todas las que deben estar se añaden otros problemas, pues a la escasa presencia de las obras de mujeres en los fondos museísticos se suma el problema de su "invisibilidad", ya que en más ocasiones de las deseables las pocas creaciones femeninas se guardan en los depósitos durante muchos años como sucede con las obras de las artistas que forman parte de los fondos del Museo del Prado, ya que en el conjunto de sus fondos hay obra de una treintena mujeres artistas, pero solo se muestran de modo permanente las obras de cuatro. Esta situación hace que en ocasiones se produzcan "descubrimientos", como sucedió con una obra de Isabel Villar que se guardó durante años en los depósitos de Es Baluart (Mallorca) siendo recientemente localizada por la directora del Centro, Nekane Aramburu. Actualmente la pintura de Isabel Villar, una de las artistas que hizo algunas interesantes obras de contenido feminista en la década de 1970, forma parte de una exposición en la que se hace una relectura crítica de los fondos del museo ${ }^{32}$. Exposición en la que además se hace especial hincapié en la aportación de las artistas en el arte más cercano en el tiempo con un apartado titulado "Procesos y contemporaneidad" cuyo hilo conductor son las obras de Ana Mendieta, Marina Abramović, Concha Jerez y Katy Bonnín. Este caso pone en evidencia la necesidad de hacerse nuevas preguntas y narraciones desde el discurso de la institución museística, así como de las posibilidades que ofrece para introducir el factor género en el museo.

En su obra Encuentros en el Museo Feminista Virtual Griselda Pollock (2010) ${ }^{33}$ plantea un proyecto de museo imaginario en el cual se producen "alianzas, relaciones y encuentros impensables entre diferentes tipos de objetos e imágenes para formular preguntas y rescatar historias desconocidas de las mujeres". Si nos preguntamos: ¿cómo sería el museo del arte español de la creación artística femenina

32 En la web del centro se dice que en esta exposición de los fondos se busca hacer un "recorrido no dogmático (en que) se intersecciona con concepciones académicas de la historia del arte para trabajar desde la fisura y el desplazamiento e incidir en el ideal de museo como gran custodio del patrimonio, su revisión y transmisión". http: //www.esbaluard.org/exposicion/coleccion-permanente-2/ (fecha de consulta: 23 de mayo de 2018)

33 Pollock, G. (2010). Encuentros en el museo feminista virtual. Madrid: Cátedra. 
que nos permitiese hacer preguntas y establecer relaciones diversas desde una óptica feminista? ¿qué nombres y que obras deberían tener presencia en él para lograr recuperar la genealogía?, nos daríamos cuenta que las ausencias y silencios de los que habla Gutiérrez Husillo (2012) ${ }^{34}$ en nuestras instituciones museísticas son aún inmensos y clamorosos.

No es pecar de pesimistas afirmar que una densa niebla se cierne aún sobre las obras de las mujeres que ahora mismo están creando arte en España. Lo más seguro es que gran parte de ellas tengan dificultades para que su obra se exponga y, si tiene la suerte de lograrlo, tiene más posibilidades de que su obra no pase a los museos y no se conserve para las generaciones futuras.

\section{Conclusiones}

Ante este panorama hemos de seguir reclamando una permanente "reinvención del museo", desarrollando nuevos relatos desde el punto de vista del género y cuestionando las políticas museísticas de nuevas adquisiciones, exposiciones, etc. que aún presentan sesgos claramente androcéntricos. Decía Adrienne Rich que "Re-visionar -el acto de volver a mirar, de ver con unos nuevos ojos, de entrar en el viejo texto desde una nueva dirección crítica- es para las mujeres más que un capítulo en la historia cultural; es un acto de supervivencia" 35 .

Uno de los objetivos de los museos ha de ser, por tanto, releer la historia del arte evitando lo que Teresa de Laurentis denomina como "puntos ciegos". Esta nueva posición permitiría desarrollar una serie de estrategias para hacer de los museos e instituciones museísticas espacios de equidad entre géneros:

1. Hay que revisar el discurso expositivo, tomando como referencia proyecto tan interesantes como Patrimonio en femenino o Museos en femenino.

2. Plantearse el porqué de las obras que están expuestas en las salas de los museos y el porqué de las que se guardan en los depósitos.

3. Revisar las políticas de adquisición de nuevos fondos desde la óptica de género.

4. Se ha de seguir reivindicando siempre que sea posible exposiciones temporales de creadoras de todas las etapas históricas (individuales y colectivas) que permitan el revisionado del patrimonio en el sentido que planteaba Adrienne Rich.

5. Se ha de potenciar el papel de los museos como mediadores y/o facilitadores entre las obras y los distintos públicos a través de los programas educativos. Programas en los que nunca ha de olvidarse que una de las preguntas claves, como dice Ana de Miguel, ha de ser: “¿y de las mujeres qué se sabe?”

Todo ello con un objetivo: conservar, exhibir, adquirir e investigar el arte hecho por mujeres, cumpliendo así con los objetivos de la LPHE establece, al tener en cuenta la creación artística en su totalidad ya que representa a más del $50 \%$ de la

\footnotetext{
34 Gutiérrez Husillo, A. (2012). Meandros de la memoria. Ausencias y silencios en torno al patrimonio en femenino. Ausencias y silencios, 14-24.

35 Rich, A. (1979). Sobre mentiras, secretos y silencios. Barcelona: Icaria.
} 
población. Sólo así lograremos en el ámbito artístico-patrimonial que en el futuro se rompa con el principio que ha venido rigiendo la historia y que tan atinadamente fue descrito por Celia Amorós: "La genealogía ha sido hasta ahora monopolio patriarcal”. 